\title{
BLUE VERSUS WHITE STIMULI IN OCULAR HYPERTENSION WITH THE FRIEDMANN MARK 1 VISUAL FIELD ANALYSER
}

\author{
CHARLES E. HUGKULSTONE and STEPHEN A. VERNON \\ Nottingham
}

\begin{abstract}
SUMMARY
Fifty eyes of fifty patients with ocular hypertension had their visual fields tested on a Friedmann Mark 1 field analyser whilst wearing a Wratten 47B blue filter in a spectacle frame. All had normal visual fields to a white stimulus. Use of a scoring system with the blue field identified 11 patients $(22 \%)$ with ocular hypertension as abnormal. The scores from this subgroup were indistinguishable from a group of subjects with early glaucomatous field loss, whilst the remaining scores were similar to normal subjects. These two subgroups of ocular hypertensive patients were similar in age and intraocular pressure. The use of a blue filter in front of the eye may offer a simple test to identify a subgroup of patients with ocular hypertension who are at increased risk of developing field loss.
\end{abstract}

Ocular hypertension $(\mathrm{OH})$ is a frequently seen condition in clinical practice. It is characterised by a raised intraocular pressure (IOP) but without an associated field defect. Longitudinal studies suggest that approximately $10 \%$ of patients with $\mathrm{OH}$ will later develop field loss, ${ }^{1,2}$ although reports of conversion to glaucoma range from $3 \%{ }^{3}$ to nearly $36 \%^{4}$

A major clinical challenge is to identify the subgroup of ocular hypertensives who will later develop field defects. This is of some importance because recent work has suggested that topical treatment can decrease the incidence of glaucomatous damage. ${ }^{5}$

The use of a blue filter with Friedmann visual field screening has been previously reported to increase the size and depth of field defects in glaucoma. ${ }^{6}$ However, later work employing a suprathreshold technique suggested that a blue stimulus was less practical and less sensitive than a white stimulus ${ }^{7}$ Both of these studies used the inbuilt filter in a Friedmann Mark 2 Visual Field Analyser. We have found that the use of a blue filter mounted in a

Correspondence to: Mr. S. A. Vernon, FRCS, FCOphth, Academic Unit of Ophthalmology, University Hospital, Nottingham NG7 2UW. spectacle frame with the Friedmann Mark 1 provided good discrimination between normal and glaucomatous patients, when used in conjunction with a scoring system based on target groups. ${ }^{8}$

This report discusses its use in ocular hypertensive subjects, and its advantages over a white stimulus.

\section{METHODS}

Patients were recruited from ophthalmic outpatient clinics at two hospitals. To be eligible for this study, unaided visual acuity had to be $6 / 18$ or better, with a best corrected acuity of at least 6/9. Known diabetics were excluded, as were patients on miotic drops. All patients gave verbal consent after the nature and purpose of this investigation had been explained to them.

Two groups of patients were identified: normal subjects, i.e. an IOP $<21 \mathrm{mmHg}$ with normal optic discs and healthy fundi and ocular hypertensives with an IOP $>21$ mmHg without field defects on standard Friedmann testing or pathological disc changes on biomicroscopy with either a fundal contact lens or a Volk 90 dioptre lens. There were no media opacities on clinical examination.

For all patients, IOPs were recorded using a standard Goldmann applanation tonometer. The diagnostic IOPs (i.e. those recorded before treatment was commenced) were used in the statistical analysis.

Two visual fields were recorded for each patient, using the Friedmann Mark 1 Visual Field Analyser. The background illumination of this machine is in the mesopic range. The protocol is described in detail elsewhere. ${ }^{8}$ Briefly, subjects were tested without spectacle correction and the threshold to a white stimulus was determined. This was defined as the filter setting when two of the four targets 2.5 degrees from fixation were seen in two of the three attempts allowed. This value was then reduced by $0.4 \mathrm{log}$ units to give the working value. The field to a white stimulus was then measured at the working value. Any points that were missed after three attempts at this level were re- 
tested with the neutral density filter being reduced in steps of $0.2 \log$ units, until the targets were seen, or the limits of the machine were reached.

The second visual field was recorded with the subject wearing a spectacle frame containing a Wratten 47B blue filter with an occluder in front of the fellow eye. This filter gives a peak transmission at 440 nanometres. The same sequence as above was employed, although the test was started using the working value already determined for the white target.

A scoring system was used with the blue field, termed the selective blue field (SBF). The highest filter setting for each target setting (groups of 2 to 4 targets) was defined as the threshold for that particular group. One point was then given for each $0.2 \log$ unit reduction from this derived 'setting threshold' for the remaining points in the group, to give the SBF score (see example in Figure 1). A similar scoring system was also used with the white field.

Statistical analysis was performed with the unpaired t-test on normally distributed data. Non-parametric tests were employed with the white and SBF scores, which were not distributed in a normal fashion. Results are given as mean (SD) unless otherwise noted. If both eyes were eligible for inclusion, only one was randomly selected for statistical analysis.

\section{RESULTS}

One hundred patients were studied, 50 normal subjects

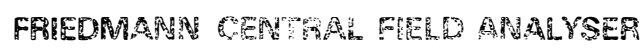

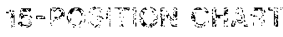

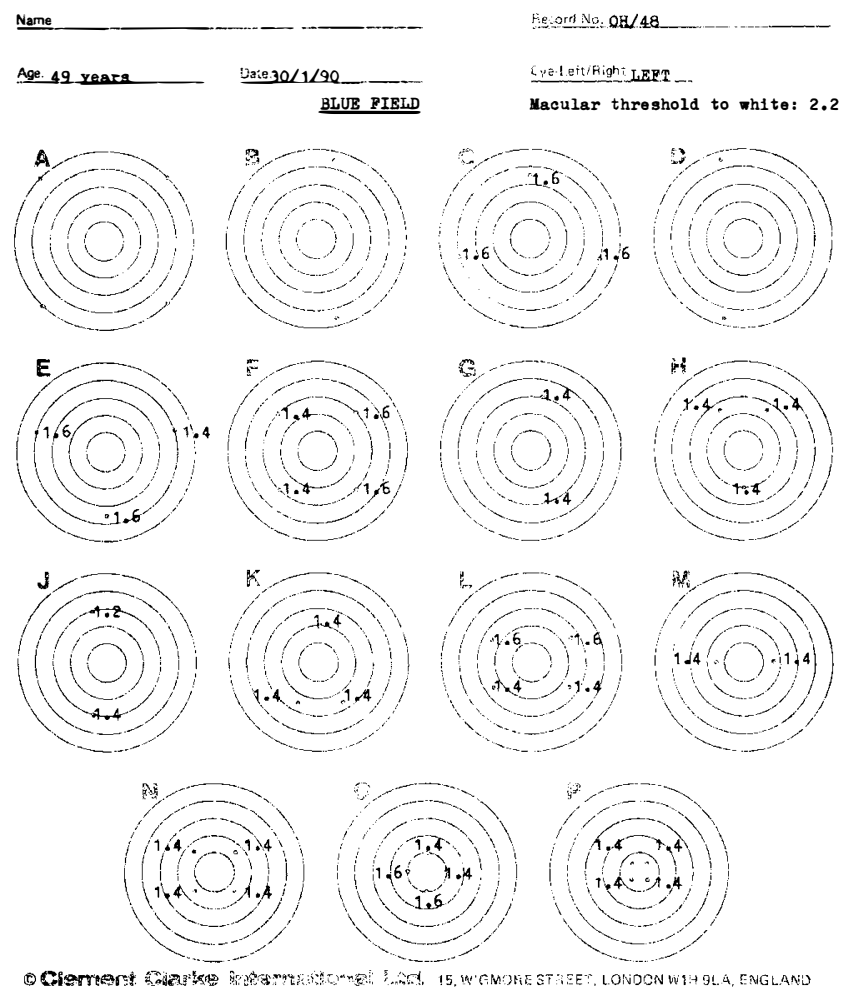

it № stoich

(a) and 50 ocular hypertensives. Their mean ages were 59.1 (10.4) and 62.0 (11.7) years respectively, which were not significantly different. The mean IOP and white and SBF scores for both groups are shown in Table I. The frequency distributions of the white and SBF scores are displayed in Figures 2 and 3.

The mean IOP of the normal subjects was, as expected, lower than the $\mathrm{OH}$ group $(\mathrm{p}<0.0001)$. There was no difference in white field score between the normal and ocular hypertensive groups, by definition. The SBF score for normal subjects was significantly lower than the $\mathrm{OH}$ group ( $\mathrm{p}>0.02)$.

There was no difference in macular thresholds between the two groups, and so the operating range of the machine was the sarne for all. This was not affected by the blue filter, because it was used at the working value calculated for the white stimulus. Thirty-six normals and 37 ocular hypertensives had both eyes tested. Paired analysis between right and left eyes (right eyes being tested first throughout) showed no differences in white and SBF scores.

An upper limit of normal (ULN) of 19.7 was calculated from the SBF scores of normal subjects, in order to give a specificity of $96 \%$ Applying this cut-off value to the $\mathrm{OH}$ group produces a low $\mathrm{OH}$ subgroup of 39 subjects and a high $\mathrm{OH}$ subgroup of 11 subjects. The number of ocular hypertensives who fall in the high $\mathrm{OH}$ subgroup is unlikely to have arisen by chance (chi squared with Yates'

\section{FREDMANA WERTRAL FIELD AMALYSE

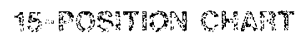
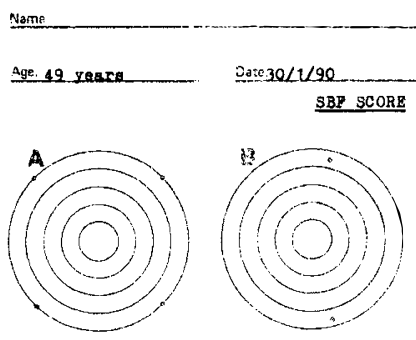

Mesori Na OH/48.
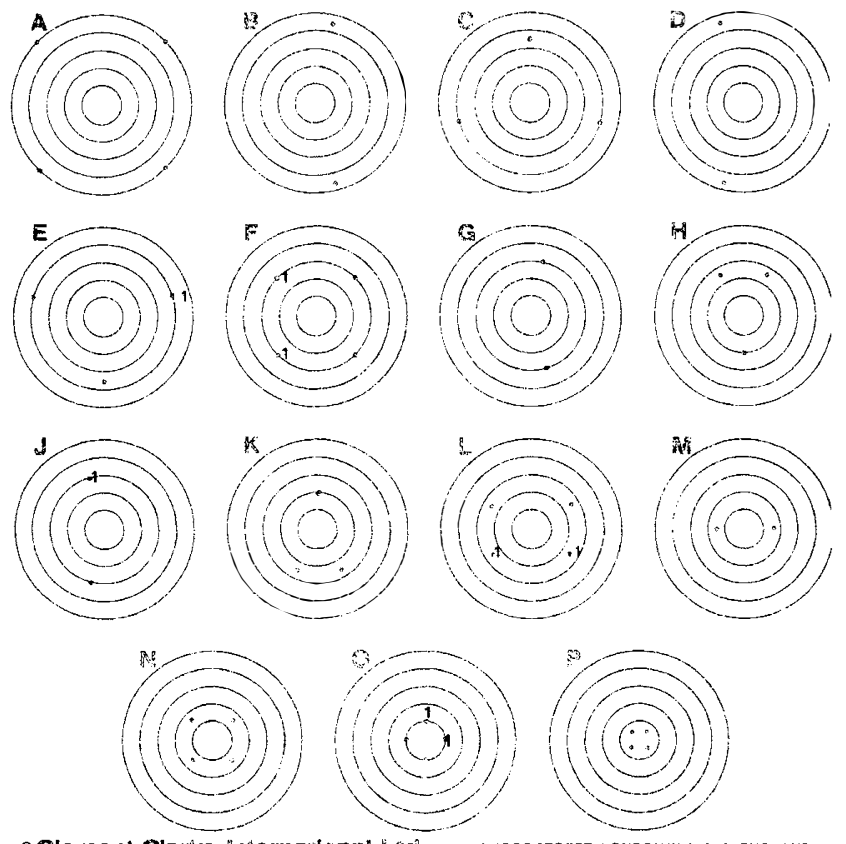

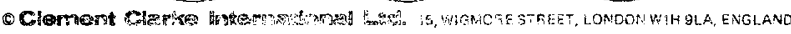

Fig. 1. (a) Visual field of left eye with the blue filter. (b) Same field, with SBF scores (see text for details). The SBF score in this example is 8 . 
Table I. Mean (SD) intra-ocular pressure (IOP) and white and selective blue field (SBF) scores

\begin{tabular}{lccc}
\hline & Normal & OH & Significance \\
\hline IOP* & $16.0(2.9)$ & $27.1(5.0)$ & $\mathrm{p}<0.0001$ \\
White field score & $0.5(1.5)$ & $0.5(1.0)$ & $\mathrm{NS}$ \\
SBF score & $7.5(6.2)$ & $12.7(10.4)$ & $\mathrm{p}<0.02$ \\
\hline
\end{tabular}

* In $\mathrm{mmHg}$

correction, $\mathrm{p}<0.02$ ). Their IOPs and white field and SBF scores are detailed in Table II. The mean age of these two subgroups was similar $(61.4(12.0)$ years and $64.2(10.5)$ years respectively, as were their IOPs.

The white field scores show no difference between the low $\mathrm{OH}$ and normal groups, whilst the high $\mathrm{OH}$ subgroup is just different from both normals and the low $\mathrm{OH}$ subgroup $(\mathrm{p}<0.05)$. There is no difference in SBF score between normals and the low $\mathrm{OH}$ group. However, the $\mathrm{SBF}$ scores of the high $\mathrm{OH}$ and normal subjects are significantly different $(\mathrm{p}<0.0001)$.

\section{DISCUSSION}

In order to perform statistical analysis on visual field data, a scoring system is required. Our system, giving one point for each $0.2 \mathrm{log}$ unit reduction from the working value, is very similar to that used by Henson and co-workers $(9,10)$. Although it gives a better indication of the depth of the defect, our system includes no attempt to weight the scoring system for clusters of missed points. Despite this, our system gives good discrimination between normal and early glaucoma subjects ${ }^{8}$ for both white and blue fields. However, some of our early glaucoma subjects had an SBF score that was less than the calculated ULN. Differing mechanisms of damage have been suggested to occur in glaucoma, supported by a study comparing the blue colour mechanism in low and high tension glaucoma. ${ }^{11}$ Sophisticated statistical analysis by Schulzer and colleagues has also demonstrated two distinct populations of glaucoma patients, one pressure sensitive and the other pressure independent. ${ }^{12}$ This may be a reason why some of our glaucoma patients gave apparently normal results with the blue filter. A recent paper has shown that some glau-

\section{NORMALS}

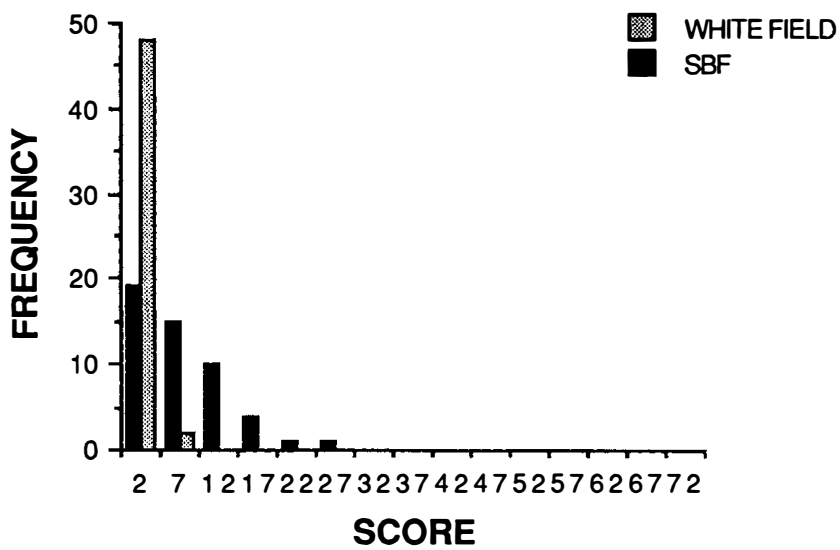

Fig. 2. Frequency histogram of white and SBF scores for normal subjects.* coma patients have preservation of normal colour vision, albeit from macular testing of colour contrast sensitivity. ${ }^{13}$ The authors of this study suggest that there may be two patterns of colour vision damage in glaucoma, one diffuse and the other sparing the macula.

As would be expected in view of the initial classification of our patients, there was no difference in white field scores between normal and ocular hypertensive subjects. The statistically significant difference in white field scores that was found with the two subgroups of ocular hypertension represents a single missed point at $0.2 \mathrm{log}$ units below the working value. Such missed points are very common in normals, ${ }^{14}$ and therefore of no clinical relevance.

Wearing the blue filter reduces both the target and background illumination to the same extent. Thus the ratio of target to background, the Weber-Fechner relation, ${ }^{15}$ remains constant. Logan and Anderson rejected the use of a blue stimulus over a white one, but kept the background illumination identical for both tests. ${ }^{7}$

Does our reduction in background illumination induce a dark adaptation factor? The lack of any asymmetry in the SBF scores in those patients who had both eyes tested indicates that any dark adaptation occurring during the test does not affect the results, providing our protocol is followed.

The advantage of the SBF score is that the use of a calculated ULN produces two subgroups from the original ocular hypertensive population, that was not possible with the IOP or the white field score. Clinical examination of the optic discs was also unhelpful in separating these two subgroups. However, recent work has emphasised the vital importance of the size of the optic disc when assessing the relevance of a particular cup-disc ratio. ${ }^{16,17}$ The routine clinical examination of the optic discs in this study could not include the disc size factor.

Yamazaki and colleagues reported a correlation between loss of blue sensitivity and the highest recorded IOP, although this was in glaucoma patients. ${ }^{18}$ This finding might be expected to have some influence on the com-

\section{OCULAR HYPERTENSIVES}

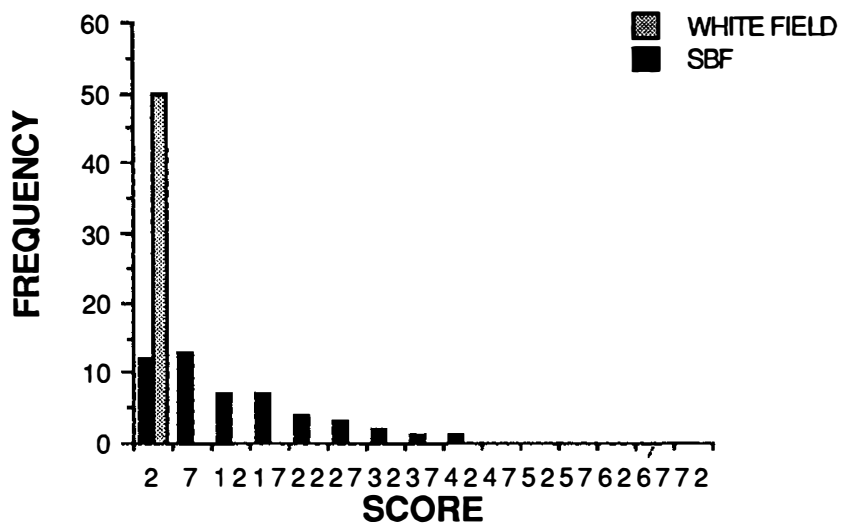

Fig. 3. Frequency histogram of white and SBF scores for ocular hypertensive subjects.*

$*$ Scores represent mid-points of groups of 5 scores e.g. $2=0.4$, $7=5.9$, etc. 
Table II. Mean (SD) intra-ocular pressure (IOP) and white and selective bue field (SBF) scores for the two subgroups of ocular hypertensives

\begin{tabular}{lrrc}
\hline & Low OH & High OH & Significance \\
\hline IOP* & $27.1(4.9)$ & $27.4(5.8)$ & NS \\
White field score & $0.3(0.8)$ & $1.2(1.5)$ & $\mathrm{p}<0.05$ \\
SBF score & $8.2(5.7)$ & $28.6(6.5)$ & $\mathrm{p}<0.0001$ \\
\hline
\end{tabular}

* In mmHg

position of the two subgroups of ocular hypertensives. However, the lack of any significant difference in the mean IOPs of the two subgroups would suggest that in fact it played no part. Interestingly, we did not find a correlation between SBF score and IOP in either group.

The low $\mathrm{OH}$ subgroup produced by use of the SBF score with a calculated ULN have similar SBF scores to normal subjects, whereas the high $\mathrm{OH}$ subgroup are indistinguishable from the results that we have previously reported in an early glaucoma group. ${ }^{8}$ The proportion of OH subjects with an abnormal SBF score changes only slightly (from $22 \%$ to $26 \%$ ) when the specificity of the test is reduced from $96 \%$ to $90 \%$. A recent study, using a blue stimulus on a yellow background, also found that $19 \%$ of ocular hypertensives had glaucomatous field defects that were not present to a white target. ${ }^{19}$ However, Sample and Weinreb reported that almost $43 \%$ of ocular hypertensives fell more than two standard deviations below normal when measuring the threshold to a blue stimulus on a yellow background. ${ }^{20}$ Contrast sensitivity has been shown to be abnormal (defined as greater than 2 SDs from agematched normals) in $63 \%$ of subjects with $\mathrm{OH}^{21}{ }^{21}$ and pattern electroretinography has also identified $63 \%$ of ocular hypertensives as being abnormal, ${ }^{22}$ with results in that subgroup being similar to those obtained from patients with early glaucoma. All of these latter proportions are somewhat greater than the reported incidence of conversion to glaucoma in longitudinal studies of ocular hypertension. ${ }^{1.4}$ However, recent work using peripheral colour contrast sensitivity ${ }^{23}$ found that about $20 \%$ of the high risk $\mathrm{OH}$ group were abnormal when using their criteria for glaucomatous subjects.

The visual fields were tested without spectacle correction. This was to prevent artefacts arising from the overlap between the subject's own spectacle frame and that of the blue filter. This may be a source of error in our results. However, whilst induced refractive errors have indeed been shown to cause a depression in thresholds values, this occurs to a similar degree across the central $25^{\circ} . .^{24}$ Thus this is unlikely to have a significant effect on the results. The mean ages of the normal and $\mathrm{OH}$ subjects were similar, so accommodation (or lack of it) would not be expected to produce the observed difference in SBF scores between these two groups. The same argument applies to the two subgroups of the $\mathrm{OH}$ group, who have similar ages to each other.

Marked loss of nerve fibres may occur before visual field defects are noted. ${ }^{25}$ This loss preferentially affects the large diameter fibres, ${ }^{26,27}$ and blue light information is pro- cessed by larger ganglion cells than in red or green.$^{28}$ Blue colour vision abnormalities have been reported in both glaucoma and some ocular hypertensives ${ }^{29.30}$ and use of a blue stimulus on a yellow background has been shown to detect glaucoma-like field defects not present with a standard white stimulus in some patients. ${ }^{19,31}$ Our simple protocol appears to detect early loss of visual function in a similar proportion of $\mathrm{OH}$ patients.

A recent study has found that topical timolol reduces the incidence of glaucomatous field loss in patients with moderate risk ocular hypertension, based on factors such as IOP and vertical cup-disc ratio. ${ }^{5}$ Their patients had similar IOPs to those in our OH group and its subgroups. Another group of investigators has also demonstrated this effect, ${ }^{32}$ although their study population had lower IOPs than our $\mathrm{OH}$ group.

Our simple adaptation to the Friedmann protocol may permit recognition of a subgroup of ocular hypertensives who will later develop glaucomatous field loss, and who should benefit from early treatment. However, longitudinal studies using automated threshold analysers are required to define the value of the use of the blue filter.

Our grateful thanks are due to Mr. A. S. Rubasingham and Mr. T. Arulampalam for kindly allowing us to study their patients.

Key words: Blue filter, Friedmann Mark I Visual Field Analyser, Glaucopma, Ocular hypertension, Visual fields.

\section{REFERENCES}

1. Kitazawa Y, Horie T, Aoki S, Suzuki M, Nishioka K: Untreated ocular hypertension. A long-term prospective study. Arch Ophthalmol 1977, 95: 1180-4.

2. David R, Livingston DG, Luntz MH: Ocular hypertension-a long-term follow-up of treated and untreated patients. Br J Ophthalmol 1977, 61: 668-74.

3. Perkins ES: The Bedford Glaucoma Survey. I. Long-term follow-up of borderline cases. Br J Ophthalmol 1973, 57: 179-85.

4. Hart WM, Yablonski M, Kass MA, Becker B: Multivariate analysis of the risk of glaucomatous visual field loss. Arch Ophthalmol 1979, 97: 1455-8.

5. Kass MA, Gordon MO, Hoff MR, Parkinson JM, Kolker AE, Hart WM Jr, Becker B: Topical timolol administration reduces the incidence of glaucomatous damage in ocular hypertensive individuals. Arch Ophthalmol 1989, 107: 1590-8.

6. Genio C and Friedmann AI: A comparison between white light and blue light on about 70 eyes of patients with early glaucoma using the Mark II visual field analyser. Doc Ophthalmol Proc Ser 1981, 26: 207-14.

7. Logan $\mathrm{N}$ and Anderson DR: Detecting early glaucomatous visual field changes with a blue stimulus. Am J Ophthalmol 1983, 95: 432-4.

8. Hugkulstone CE and Vernon SA: The use of a blue filter in visual field analysis. Br J Ophthalmol 1991, 75: 155-7.

9. Henson DB and Dix SM: Evaluation of the Friedmann Visual Field Analyser Mark II. Part 2. Results from a population with induced field defects. Br J Ophthalmol 1984, 68: 463-7.

10. Henson D, Hobley A, Chauhan B, Sponsel W, Dallas N: Importance of visual field score and asymmetry in the detection of glaucoma. Am J Optom Physiol Opt 1986, 63: 714-23.

11. Yamazaki Y, Lakowski R, Drance SM: A comparison of the 
blue color mechanism in high- and low-tension glaucoma. Ophthalmology 1989, 96: 12-5.

12. Schulzer M, Drance SM, Carter CJ, Brooks DE, Douglas GR, Lau W: Biostatistical evidence for two distinct chronic open angle glaucoma populations. $\mathrm{Br} J$ Ophthalmol 1990,74: 196-200.

13. Falcao-Reis FM, O'Sullivan F, Spileers W, Hogg C, Arden GB: Macular colour contrast sensitivity in ocular hypertension and glaucoma: evidence for two types of defect. BrJ Ophthalmol 1991, 75: 598-602.

14. Henson DB, Dix SM, Oborne AC: Evaluation of the Friedmann Visual Field Analyser Mark II. Part 1. Results from a normal population. Br J Ophthalmol 1984, 68: 458-62.

15. Hart WM Jr. Visual adaptation. In Moses RA and Hart WM Jr, eds. Adler's Physiology of the eye. Clinical Application. C.V. Mosby. St Louis. 1987: 389-414.

16. Britton RJ, Drance SM, Schulzer M, Douglas GR, Mawson DK: The area of the neuroretinal rim of the optic nerve in normal eyes. Am J Ophthalmol 1987, 103: 497-504.

17. Quigley HA, Brown AE, Morrison JD, Drance SM: The size and shape of the optic disc in normal human eyes. Arch Ophthalmol 1990, 108: 51-7.

18. Yamazaki Y, Drance SM, Lakowski R, Schulzer M: Correlation between color vision and highest intraocular pressure in glaucoma patients. Am J Ophthalmol 1988, 106: 397-9.

19. Heron G, Adams AJ, Husted R: Central visual fields for short wavelength sensitive pathways in glaucoma and ocular hypertension. Invest Ophthalmol Vis Sci 1988, 29: 64-72.

20. Sample PA and Weinreb RN: Color perimetry for assessment of primary open-angle glaucoma. Invest Ophthalmol Vis Sci 1990, 31: 1869-75.

21. Ross JE, Bron AJ, Reeves BC, Emmerson PG: Detection of optic nerve damage in ocular hypertension. BrJ Ophthalmol 1985, 69: 897-903.

22. Weinstein GW, Arden GB, Hitchings RA, Ryan S, Calthorpe CM, Odom JV: The pattern electroretinogram (PERG) in ocular hypertension and glaucoma. Arch Ophthalmol 1988, 106: $923-8$.
23. Yu TC, Falcao-Reis F, Spileers W, Arden GB: Peripheral color contrast. A new screening test for preglaucomatous visual loss. Invest Ophthalmol Vis Sci 1991, 32: 2779-89.

24. Heuer DK, Anderson DR, Feuer WJ, Gressel MG: The influence of refraction accuracy on automated perimetric threshold measurements. Ophthalmology 1987, 94: 1550-53.

25. Quigley HA, Addicks EM, Green WR: Optic nerve damage in human glaucoma. III. Quantitative correlation of nerve fiber loss and visual field defect in glaucoma, ischemic neuropathy, papilledema, and toxic neuropathy. Arch Ophthalmol 1982, 100: 135-46.

26. Quigley HA, Sanchez RM, Dunkelberger GR, L'Hernault NL, Baginski TA: Chronic glaucoma selectively damages large optic nerve fibers. Invest Ophthalmol Vis Sci 1987, 28: 913-20.

27. Quigley HA, Dunkelberger GR, Green WR: Chronic human glaucoma causing selectively greater loss of large optic nerve fibers. Ophthalmology 1988, 95: 357-63.

28. De Monasterio FM: Asymmetry of on- and off-pathways of blue-sensitive cones of the retina of macaques. Brain Res 1979, 166: 39-48.

29. Drance SM, Lakowski R, Schulzer M, Douglas GR: Acquired color vision changes in glaucoma. Use of 100-Hue test and Pickford anomaloscope as predictors of glaucomatous field change. Arch Ophthalmol 1981, 99: 829-31.

30. Gündüz K, Arden GB, Perry S, Weinstein GW, Hitchings RA: Colour vision defects in ocular hypertension and glaucoma. Quantification with a computer-driven color television system. Arch Ophthalmol 1988, 106: 929-35.

31. Hart WM Jr, Silverman SE, Trick GL, Nesher R, Gordon MO: Glaucomatous visual field damage. Luminance and color-contrast sensitivities. Invest Ophthalmol Vis Sci 1990, 31: 359-67.

32. Epstein DL, Krug JH Jr, Hertzmark E, Remis LL and Edelstein DJ: A long-term clinical trial of timolol therapy versus no treatment in the management of glaucoma suspects. Ophthalmology 1989, 96: 1460-7. 